\title{
Modeling Channel Conflict Probabilities between IEEE 802.15 based Wireless Personal Area Networks
}

\author{
Ling-Jyh Chen \\ Institute of Information Science \\ Academia Sinica \\ cclljj@iis.sinica.edu.tw
}

\begin{abstract}
With the increasingly deployed Wireless Personal Area Network (WPAN) devices, channel conflict has become very frequent and severe when one WPAN technology coexists with other WPAN technologies in the same interfering range. In this paper, we study the coexistence issue between various IEEE 802.15 based WPAN technologies. We present analytical models on the non-conflicting channel allocation probabilities, focusing on the coexistence scenarios of one WPAN technology coexisting with another. The results show that channel allocation conflicts occurs frequently in all cases, and is especially severe between IEEE 802.15.3 and IEEE 802.15.4 networks. On the other hand, the probability of non-conflict channel allocation is less dramatic between a single IEEE 802.15.1 and coexisting IEEE 802.15.4 networks. In addition, the proposed models in this paper are also applicable to other wireless technologies, as long as the channel allocation mechanisms are known.
\end{abstract}

\section{INTRODUCTION}

Wireless Personal Area Network (WPAN) technologies have fueled the development as well as the wide proliferation of wireless personal devices (e.g. PDAs, Bluetooth headset, PSP, and etc). Yet, the popularity of these wireless devices have resulted in many forms of frequency spectrum clash amongst the different wireless technologies. To understand the performance of these wireless devices in different interference situations, it is increasingly important to study the coexistence issue amongst the existing wireless technologies.

Various wireless technologies have been developed for WPAN purposes. For instance, Bluetooth [1] (as described by the IEEE 802.15.1 standard [2]) has been proposed as a cable replacement technology for wireless personal devices. IEEE 802.15.3 standard [3] has been proposed for High Rate WPAN (HR-WPAN) applications, and IEEE 802.15.4 standard [4] has been drafted for Low Rate WPAN (LR-WPAN) uses.

Since IEEE 802.15.1, IEEE 802.15.3, and IEEE 802.15.4 all operate in the same $2.4 \mathrm{GHz}$ ISM (Industrial-ScientificMedical) frequency band, channel allocation conflicts are inevitable between these WPAN technologies. The coexistence issues will become even severe while these WPAN technologies also coexist with other $2.4 \mathrm{GHz}$ based wireless/radio technologies (e.g. IEEE 802.11b/g [5], cordless phone, and microwave oven). It soon becomes important to understand

\author{
Tony Sun, Mario Gerla \\ Computer Science Department \\ University of California at Los Angeles \\ \{tonysun,gerla\}@cs.ucla.edu
}

TABLE I

WIRELESS TECHNOLOGIES IN 2.4GHZ ISM FREQUENCY BAND.

\begin{tabular}{|c|c|c|c|c|}
\hline IEEE Standard & $802.11 \mathrm{~b} / \mathrm{g}$ & 802.15 .1 & 802.15 .3 & 802.15 .4 \\
\hline Frequency Band & $2.4 \mathrm{GHz}$ & $2.4 \mathrm{GHz}$ & $2.4 \mathrm{GHz}$ & $2.4 \mathrm{GHz}$ \\
\hline rcv Bandwidth & $22 \mathrm{MHz}$ & $1 \mathrm{MHz}$ & $15 \mathrm{MHz}$ & $2 \mathrm{MHz}$ \\
\hline Number of Channels & 11 & 79 & 5 & 16 \\
\hline Max Rate (Mbps) & $11 / 54$ & 0.72 & 55 & 0.25 \\
\hline Transmission Range & $100 \mathrm{~m}$ & $10 \mathrm{~m}$ & $10 \mathrm{~m}$ & $20 \mathrm{~m}$ \\
\hline Applications & WLAN & WPAN & HR-WPAN & LR-WPAN \\
\hline
\end{tabular}

the characteristics of each channel allocation scheme and how each channel allocation scheme interacts with the others. Table I summarizes some of the relevant properties of the wireless standards mentioned above.

The coexistence issue between WPAN and other wireless technologies has been extensively studied. [6] first performed a systematical study on the coexistence of IEEE $802.11 \mathrm{~b}$ and Bluetooth devices via testbed measurements, and [7] has studied the coexistence of Bluetooth and microwave ovens by measuring the channel error rates of Bluetooth devices. In addition, analytical models for the coexistence of Bluetooth and IEEE 802.11b have also been proposed in [8][9][10] at various configuration scenarios, and [11] has proposed an analytical model to evaluate the coexistence issue between IEEE 802.15.4 and IEEE 802.11b networks. Moreover, the discussion on the coexistence issue between IEEE 802.11 and the IEEE 802.15 based WPAN technologies has been included in IEEE standards. For instance, the IEEE 802.15.2 standard [12] addressed the coexistence of IEEE 802.15.1 and IEEE $802.11 \mathrm{~b}$ networks. [3] and [4] also provided some evaluation on the coexistence issues between IEEE 802.15.3/4 and IEEE $802.11 \mathrm{~b}$ networks at various distances.

Nonetheless, previous analytical work assumed operating channels are already conflicted when modeling error rates between different wireless technologies, and they focused primarily on the coexistence between WPAN and other technologies (e.g. WLAN and microwave ovens). The coexistence issues between various WPAN technology were still not fully explored.

In this paper, we study the coexistence issues between 
various IEEE 802.15 based WPAN technologies. We present analytical models to calculate the channel collision probability between two different WPAN technologies. The results demonstrate that channel collision occurs frequently in almost all cases, and is especially severe between IEEE 802.15.3 and IEEE 802.15.4 networks. On the other hand, the channel collision probability is much more amiable between a single IEEE 802.15.1 and other coexisting IEEE 802.15.4 networks. Moreover, the models presented in this paper are also useful in calculating the channel collision probability for other applicable wireless technologies (as long as their channel allocation mechanisms are known).

The rest of the paper is organized as follows. In section II, we present an brief overview of the various WPAN technologies and their channel allocation schemes. In section III, we present analysis on the probability of channel collision between different pairing of wireless technologies. Finally, section IV concludes the paper.

\section{OVERVIEW OF WPAN TECHNOLOGIES}

In this section, we present an overview of the IEEE 802.15 based WPAN technologies with focuses on the channel allocation schemes.

\section{A. IEEE 802.15.1}

IEEE 802.15.1 [2] is a WPAN standard based on the Bluetooth v1.1 Specification [1], which is a short-range radio technology operating in the unlicensed $2.4 \mathrm{GHz}$ ISM frequency band. The original goal of Bluetooth was to replace the numerous proprietary cables to provide a universal interface for devices to communicate with each other. However, it soon became to use Bluetooth technology to interconnect various Bluetooth devices to form so-called personal area networks [13], and facilitate more creative ways of exchanging data. Low cost and smaller footprint of Bluetooth chips consequently met with high demands.

IEEE 802.15.1 employs FHSS (Frequency Hopping Spread Spectrum) technique. There are 79 hopping frequencies ${ }^{1}$, each having a bandwidth of $1 \mathrm{MHz}$. The 79 hopping frequencies are:

$$
f=2402+k ; k=0 \ldots 78
$$

The frequency hopping sequence is determined by a hopping kernel. In each time, the hopping kernel first selects a segment of 64 adjacent channels (note that the last channel, i.e. $k=78$, is adjacent to the first channel, i.e. $k=0$, as illustrated in Figure 1) and then hops to 32 of them without repetition in a random order. Next, a different 32-hop sequence is chosen from another segment of 64 adjacent channels, and etc. As a result, a pseudo-random sequence of frequency hopping slides through the 79 available channels.

It should also be mentioned that, in addition to the basic hopping kernel defined in the IEEE 802.15.1 standard, a new Adaptive Frequency Hopping (AFH) kernel has been proposed

\footnotetext{
${ }^{1}$ In some countries, e.g. France, there are only 23 hopping frequencies available
}

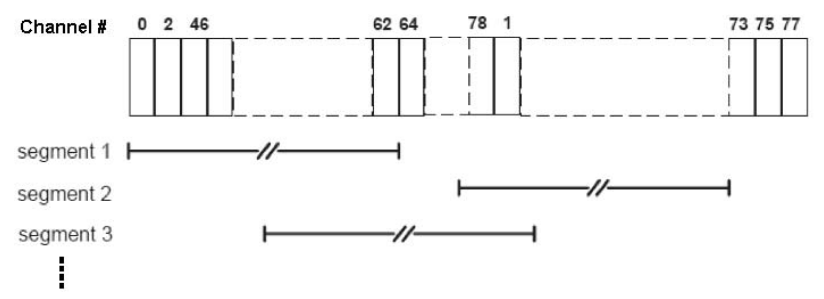

Fig. 1. An example of the sequence selection of IEEE 802.15.1 frequency hopping.

in the follow-up Bluetooth spec [14][15]. In AFH, the 79 channels are classified into two groups. One of them is the group of channels which shall be unused (i.e. these channels might have been heavily interfered), and the other is the group of channels which should be used. Using the basic hopping kernel, if the selected channel belongs to the unused group, AFH employs a mapping function, which uniformly maps unused channels to used channels, to replace the selected channel to a used channel. As a result, only used channels will be used in AFH, and the unused channels are avoided.

Though AFH is promising in alleviating interference to Bluetooth networks, we do not include AFH in our evaluation since it is not specified in IEEE 802.15.1 standard yet. We intend to evaluate the channel confliction issue with AFH in the near future.

\section{B. IEEE 802.15.3}

IEEE 802.15.3 [3] is designed to facilitate High-Rate Wireless Personal Area Networks (HR-WPAN) for fixed, portable, and moving devices within a personal operating space. The main purpose of IEEE 802.15.3 is to provide low cost, low complexity, low power consumption, and high data rate connectivity for wireless personal devices. Thus, it is designed to support at least $11 \mathrm{Mbps}$ data rate within at least 10 meters range ${ }^{2}$.

The IEEE 802.15 .3 standard is operated in $2.4 \mathrm{GHz}$ ISM frequency band. Unlike IEEE 802.15.1, which employs FHSS on PHY layer, IEEE 802.15.3 uses Direct Sequence Spread Spectrum (DSSS), and it does not allow changes of operating channels once a connection is initiated.

There are two sets of channels available for IEEE 802.15.3 operation. The first set is the high-density mode which consists of 4 channels, and the second set is the IEEE $802.11 \mathrm{~b} \mathrm{co-}$ existence mode which occupies 3 channels (i.e. the center frequency of each channel is the same as in IEEE 802.11b). For both of them, the channel bandwidth is $15 \mathrm{MHz}$ for each IEEE 802.15.3 channel. Since the two outer channels of the two sets are overlapped, there are totally 5 channels allowed for IEEE 802.15.3 operation. Table II describes the channel allocation of IEEE 802.15.3 standard. In this paper, we will concentrate our analysis on the second set of channels (i.e. $802.11 \mathrm{~b}$ coexistence mode).

\footnotetext{
${ }^{2}$ There is an alternative 802.15.3 PHY, namely IEEE 802.15.3a [16] (a.k.a. Ultra Wideband), supporting at least $110 \mathrm{Mbps}(400 \mathrm{Mbps})$ data rate within $10 \mathrm{~m}(5 \mathrm{~m})$ transmission range.
} 


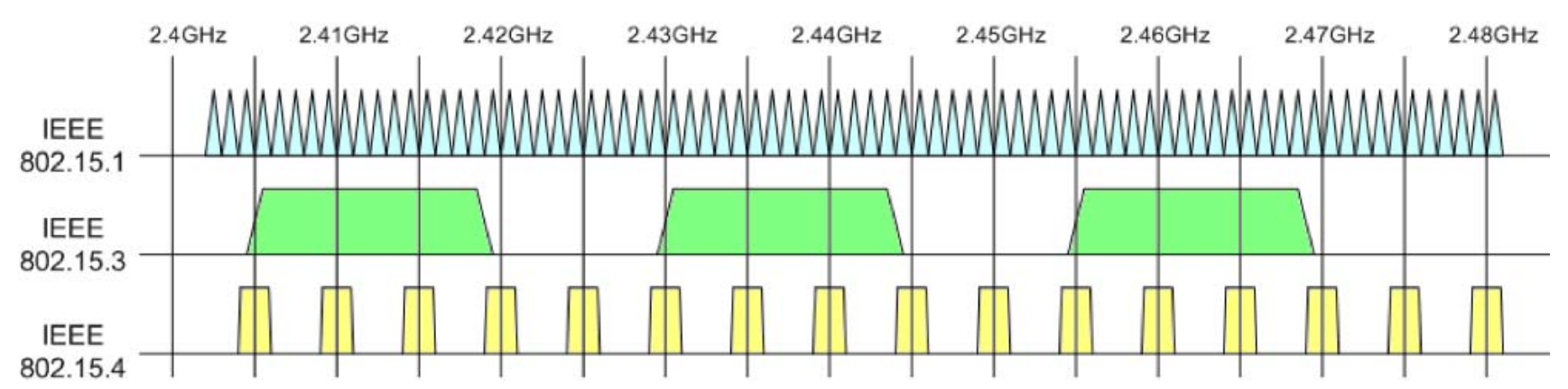

Fig. 2. Channel allocations of IEEE 802.15 based WPAN technologies.

TABLE II

IEEE 802.15.3 CHANNEL ALLOCATION.

\begin{tabular}{|c|c|c|c|}
\hline Channel & Center Frequency & High-density & $802.11 \mathrm{~b}$ coexistence \\
\hline 1 & $2.412 \mathrm{GHz}$ & $\sqrt{ }$ & $\sqrt{ }$ \\
\hline 2 & $2.428 \mathrm{GHz}$ & $\sqrt{ }$ & $\sqrt{ }$ \\
\hline 3 & $2.437 \mathrm{GHz}$ & & $\sqrt{ }$ \\
\hline 4 & $2.445 \mathrm{GHz}$ & $\sqrt{ }$ & \\
\hline 5 & $2.462 \mathrm{GHz}$ & $\sqrt{ }$ & \\
\hline
\end{tabular}

\section{IEEE 802.15.4}

IEEE 802.15.4 [4] addresses the needs of Low-Rate Wireless Personal Area Networks (LR-WPAN). While other WLAN (e.g. IEEE 802.11.a/b/g [5]) and WPAN (e.g. IEEE 802.15.1 and 802.15.3) technologies focus on providing high data throughput over wireless ad hoc networks, IEEE 802.15.4 is designed to facilitate those wireless networks, which are mostly static, large, and consuming small bandwidth and power. Therefore, the IEEE 802.15.4 technology is anticipated to enable various applications in the fields of home networking, automotive networks, industrial networks, interactive toys and remote metering.

IEEE 802.15.4 employs DSSS on PHY layer, and it is operated in three frequency bands. Among a total of 27 channels (with $2 \mathrm{MHz}$ width for each channel) across these three bands, sixteen channels are available in the $2.4 \mathrm{GHz}$ band with $250 \mathrm{kbps}$ maximum data throughput, 10 in the $915 \mathrm{MHz}$ band with $40 \mathrm{kbps}$ maximum data throughput, and 1 in the $868 \mathrm{MHZ}$ band with 20 kbps maximum data throughput. The center frequency of these channels is defined as follows:

$$
f= \begin{cases}868.3 & ; k=0 \\ 906+2(k-1) & ; k=1 \ldots 10 \\ 2405+5(k-11) & ; k=11 \ldots 26\end{cases}
$$

Figure 2 illustrates the channel allocation of the three IEEE 802.15 based WPAN technologies discussed.

\section{ANALYSIS}

In this section, we present analysis on the non-conflicting channel allocation probability, i.e. $P_{\text {good }}$, between the different IEEE 802.15 based WPAN technologies. Note that we do not consider scenarios consisting of multiple IEEE 802.15.1 networks, since such scenarios can be solved by combining our analysis and previous studies [17].

\section{A. 802.15.1 with one or more 802.15.3 networks}

Here, we model the probability of non-conflicting channel allocation probability $\left(P_{\text {good }}\right)$ when an IEEE 802.15 .1 network coexists with $n$ IEEE 802.15 .3 networks $(802.11 \mathrm{~b}$ coexistence mode). For simplicity, we assume the employed channels of the $n$ IEEE 802.15.3 networks are not conflicted.

We define random variable $R$ as the number of conflicting channels in the selected 64 adjacent segment in IEEE 802.15.1, and $K$ as the number of conflicting channels in the selected 32-hop channel sequence (out of the $R$-conflicted segment). The random variable $S$ denotes the channel status (0: no channel allocation conflict occurred; 1: channel allocation conflict occurred). We define $P_{\text {good }}=P[S=0]$ and study the coexistence issues in three cases as follows.

1) Case 1: $n=1$

From Figure 2, when there is only one coexisting IEEE 802.15.3 network, one can obtain $P[R=r]$, i.e. the probability such that there are $r$ conflicting channels in the selected segment of 64-adjacent channels (via counting), and obtain

$$
P[R=r]=\left\{\begin{array}{cl}
\frac{49}{79} & ; r=16 \\
\frac{2}{79} & ; 1 \leq r \leq 15 \\
0 & ; \text { otherwise }
\end{array}\right.
$$

Given $r$ conflicted channels in the 64 adjacent channels, the probability of $k$ channel conflict out of the selected 32-hop sequence $(k \leq r)$ is obtained by:

$$
P[K=k \mid R=r]=\frac{32 ! 32 !}{64 !}\left(\begin{array}{l}
r \\
k
\end{array}\right)\left(\begin{array}{l}
64-r \\
32-k
\end{array}\right)
$$

In addition, given $k$ channel conflict in the selected 32-hop sequence, the probability of channel conflict while selecting one channel from the 32-hop sequence is defined as:

$$
P[S=1 \mid K=k, R=r]=\frac{k}{32}
$$

To sum up all conditions of possible $r$ and $k$ values (shown in Eq. 6 and 7), one can obtain $P_{\text {good }}$, i.e. the probability of no channel confliction, by Eq. 8, and the non-conflicting channel allocation probability is around 0.8 . 
$P[S=1 \mid R=r]=\sum_{k=1}^{r}(P[S=1 \mid K=k, R=r] P[K=k \mid R=r])$

$$
\begin{aligned}
P[S=1] & =\sum_{r=1}^{16}(P[S=1 \mid R=r] P[R=r]) \\
& \approx 0.202532 \\
P_{\text {good }} & =1-P[S=1] \approx 0.797468
\end{aligned}
$$

2) Case 2: $n=2$

When there are two distinct IEEE 802.15.3 networks $(n=$ 2 ) occupying two distinct channels, either channel $(1,3)$, (3, $5)$, or $(1,5)$ is utilized. The $P[R=r]$ of these three cases are shown in Eq. 9, 10 and 11. We assume these three cases are uniformly distributed. Therefore, the overall $P[R=r]$ is obtained by summing up the three cases as shown in Eq. 12 .

(a) channel $(1,3)$ are utilized

$$
P_{a}[R=r]=\left\{\begin{array}{cl}
\frac{24}{79} & ; r=32 \\
\frac{2}{79} & ; 27 \leq r \leq 31 \\
\frac{9}{79} & ; r=26 \\
\frac{4}{79} & ; 17 \leq r \leq 25 \\
0 & ; \text { otherwise }
\end{array}\right.
$$

(b) channel $(3,5)$ are utilized

$$
P_{b}[R=r]=\left\{\begin{array}{cl}
\frac{24}{79} & ; r=32 \\
\frac{2}{79} & ; 27 \leq r \leq 31 \\
\frac{9}{79} & ; r=26 \\
\frac{4}{79} & ; 17 \leq r \leq 25 \\
0 & ; \text { otherwise }
\end{array}\right.
$$

(c) channel $(1,5)$ are utilized

$$
\begin{aligned}
& P_{c}[R=r]=\left\{\begin{array}{cl}
\frac{20}{79} & ; r=32 \\
\frac{2}{79} & ; r=31 \\
\frac{5}{79} & ; r=30 \\
\frac{4}{79} & ; 17 \leq r \leq 29 \\
0 & ; \text { otherwise }
\end{array}\right. \\
& P[R=r]=\frac{1}{3} P_{a}[R=r]+\frac{1}{3} P_{b}[R=r]+\frac{1}{3} P_{c}[R=r] \\
& =\left\{\begin{array}{cl}
\frac{68}{237} & ; r=32 \\
\frac{2}{79} & ; r=31 \\
\frac{3}{79} & ; r=30 \\
\frac{8}{237} & ; 27 \leq r \leq 29 \\
\frac{22}{237} & ; r=26 \\
\frac{4}{79} & ; 17 \leq r \leq 25 \\
0 & ; \text { otherwise }
\end{array}\right.
\end{aligned}
$$

Similar to the analysis presented in Case 1, one can then obtain the probability of non-conflicting channel allocation for $n=2$ case, as shown in Eq. 13 and 14. The probability turns out to be around 0.6. Clearly, channel conflict are very common in this scenario.

$$
\begin{gathered}
P[S=1]=\sum_{r=17}^{32}(P[S=1 \mid R=r] P[R=r]) \\
P_{\text {good }}=1-P[S=1] \approx 0.594937
\end{gathered}
$$

3) Case 3: $n=3$

When there are three distinct IEEE 802.15.3 networks ( $n=$ 3 ) occupying all three distinct channels (channel 1, 3, and 5 are all utilized by the IEEE 802.15.3 networks). $P[R=r]$ in this scenario is then calculated and shown in Eq. 15. The non-conflicting channel allocation probability, $P_{\text {good }}$, is then obtained using the same method as in the previous cases, and it turns out to be less than 0.4 in this case. Unsurprisingly, the results show that the probability for channel conflict is much higher (or more frequent) in this scenario compare to the case where $n=2$.

$$
\begin{gathered}
P[R=r]=\left\{\begin{array}{cl}
\frac{3}{79} & ; r=46 \\
\frac{2}{79} & ; 43 \leq r \leq 45 \\
\frac{16}{79} & ; r=42 \\
\frac{6}{79} & ; 33 \leq r \leq 41 \\
0 & ; \text { otherwise }
\end{array}\right. \\
P[S=1]=\sum_{r=33}^{46}(P[S=1 \mid R=r] P[R=r]) \\
P_{\text {good }}=1-P[S=1] \approx 0.392405
\end{gathered}
$$

\section{B. 802.15.1 with one or more 802.15.4 networks}

For an IEEE 802.15.1 network coexisting with multiple IEEE 802.15.4 networks, we also model the non-conflicting channel allocation probability $\left(P_{\text {good }}\right)$ for various number of distinct IEEE 802.15.4 networks. The same random variables (i.e. $R, K$, and $S$ ) are also used in the analysis for consistency with the previous subsection. We present the analysis in two cases as follows.

\section{1) Case 1: $n=1$}

Since an IEEE 802.15.4 network occupies at most two IEEE 802.15.1 channels, when there only exists one IEEE 802.15.4 network, $P[R=r]$ can be calculated as

$$
P[R=r]=\left\{\begin{array}{cl}
\frac{63}{79} & ; r=2 \\
\frac{2}{79} & ; r=1 \\
\frac{14}{79} & ; r=0 \\
0 & ; \text { otherwise }
\end{array}\right.
$$

Similarly, using Eq. 6 and 7, we derive the non-conflicting channel allocation probability $\left(P_{\text {good }}\right)$ for the case where $n=$ 1 with Eq. 20. $P_{\text {good }}$ turned out to be around 0.97 , which is much greater than in the case of one IEEE 802.15.1 network coexisting with one IEEE 802.15.3 network. Intuitively, this means that a single IEEE 802.15.1 network coexists much better with a single IEEE 802.15.4 network than with an IEEE 802.15.3 network. This result also instinctively makes sense, since the received bandwidth of IEEE 802.15.4 is 7 time narrower compared to IEEE 802.15.3. 


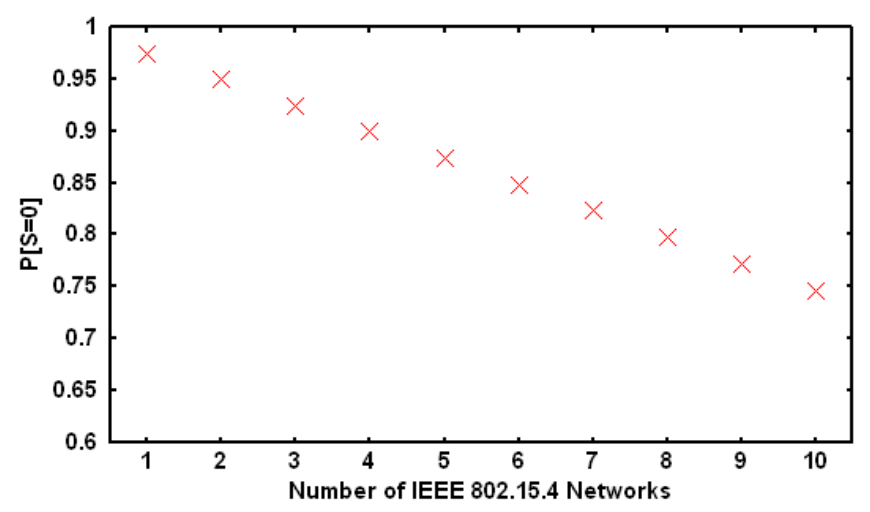

Fig. 3. Simulation results of $P[S=0]$ while one IEEE 802.15.1 network coexists with multiple IEEE 802.15.4 networks.

$$
\begin{gathered}
P[S=1]=\sum_{r=1}^{2}(P[S=1 \mid R=r] P[R=r]) \\
P_{\text {good }}=1-P[S=1] \approx 0.974684
\end{gathered}
$$

2) Case 2: $n>1$

We then perform Monte Carlo simulation to evaluate $P_{\text {good }}$ for the case where a single IEEE 802.15.1 network coexists with multiple IEEE 802.15.4 networks $(n=1 \ldots 10)$. The results are shown in Fig. 3.

The results clearly show that $P_{\text {good }}$ decreases (almost linearly) as the number of the coexisting IEEE 802.15.4 networks increases. More specifically, While $n<3$, more than $90 \%$ IEEE 802.15.1 channel allocations will not cause any channel conflicts. However, when $n$ becomes larger than 7, more than $20 \%$ of the channel allocations are conflicted. In any case, $P_{\text {good }}$ is still much higher than compare to the amount of channel conflict exist between IEEE 802.15.1 and IEEE 802.15 .3 networks.

\section{802.15.3 with one or more 802.15.4 networks}

Since both IEEE 802.15.3 and IEEE 802.15.4 technologies employ DSSS technique, they operate on one selected channel once the connection is initiated. Therefore, the non-conflict channel allocation probability $\left(P_{\text {good }}\right)$ between a single IEEE 802.15.3 network coexisting with multiple IEEE 802.15.4 networks can be easily obtained by applying the well-known Pigeonhole Principle [18]. More specifically, Since one IEEE 802.15.3 channel overlaps four IEEE 802.15.4 channels and there are totally 16 IEEE 802.15 .4 channels, it's appearent that one IEEE 802.15.3 network can coexist with at most 12 IEEE 802.15 .4 networks, and $P_{\text {good }}$ can be modeled by:

$$
P_{\text {good }}=\left\{\begin{array}{cl}
\frac{(16-n) !}{16 !} \times \frac{12 !}{(12-n) !} & ; 0 \leq n \leq 12 \\
0 & ; \text { otherwise }
\end{array}\right.
$$

Figure 4 illustrates the relationship of $P_{\text {good }}$ and the number of coexisting IEEE 802.15.4 networks for one IEEE 802.15.3 network. The results show that $P_{\text {good }}$ is always below 0.8

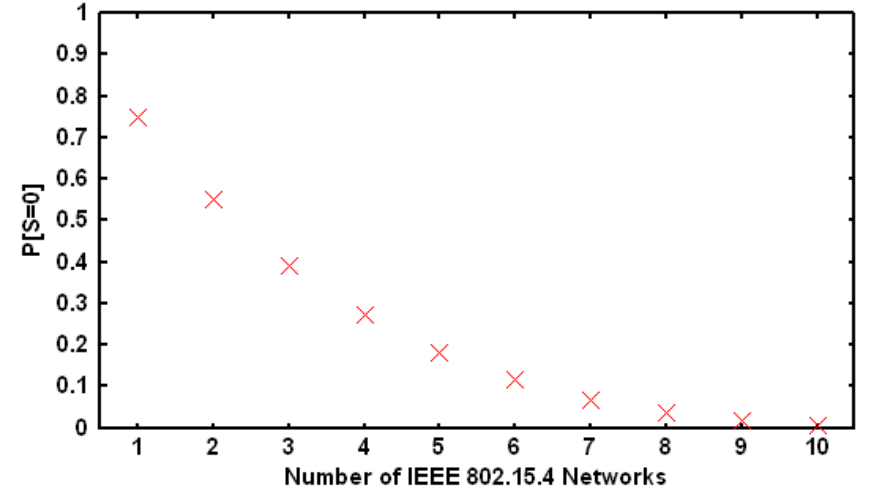

Fig. 4. Simulation results of $P[S=0]$ while one IEEE 802.15.3 network coexists with multiple IEEE 802.15 .4 networks.

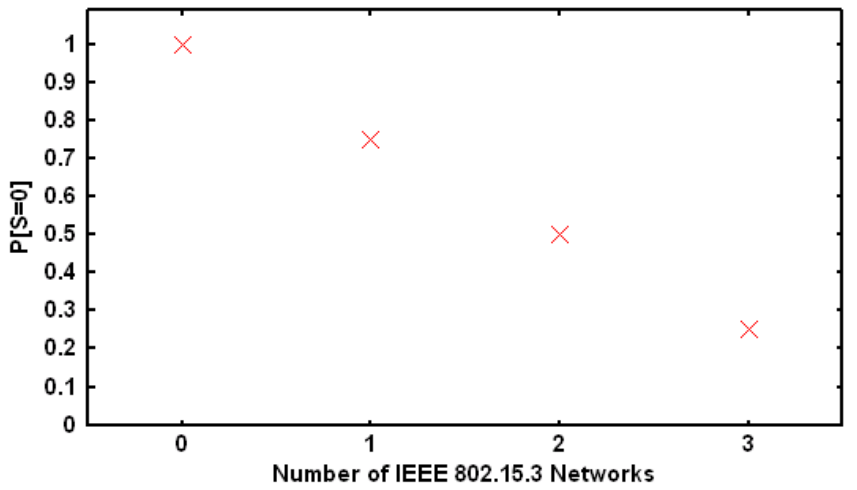

Fig. 5. Simulation results of $P[S=0]$ while one IEEE 802.15.4 network coexists with multiple IEEE 802.15.3 networks.

as long as there exist coexisting IEEE 802.15.4 networks. $P_{\text {good }}$ decreases nearly exponentially as $n$ increases, and $P_{\text {good }}$ becomes less than 0.5 when $n$ is larger than 2 . It turns out that IEEE 802.15.3 may not coexist well with multiple IEEE 802.15.4 networks, since channel conflicts occurs very frequently.

\section{802.15.4 with one or more 802.15.3 networks}

In the last scenario, when one IEEE 802.15.4 network coexists with $n$ IEEE 802.15.3 networks, there are two possible cases: a) the IEEE 802.15.4 network operates on one of the four non-overlapped channels (i.e. the IEEE 802.15.4 channels are not overlapped with IEEE 802.15.3 channels, as shown in Figure 2); b) the IEEE 802.15.4 network operates on one of the overlapped channels.

In the first case, the probability of non-conflicting channel allocation is always 1 regardless of the number of coexisting IEEE 802.15.3 networks. Whereas in the second case, the probability of non-conflicting channel allocation is $\frac{3-n}{3}$. By un-conditioning the two cases, the $P_{\text {good }}$ for a single IEEE 802.15.4 network coexisting with $n$ IEEE 802.15.3 networks can be calculated by: 


$$
P_{\text {good }}=\left\{\begin{array}{cl}
\frac{4}{16}+\frac{12}{16} \times \frac{3-n}{3} & ; 0 \leq n \leq 3 \\
0 & ; \text { otherwise }
\end{array}\right.
$$

Figure 5 illustrates the relationship of $P_{\text {good }}$ and the number of coexisting IEEE 802.15.3 networks for a single IEEE 802.15.4 network, which shows $P_{\text {good }}$ decreases linearly as $n$ increases. Similar to the results presented in the previous subsection, the results indicate that channel conflicts occur more frequently as the number of coexisting IEEE 802.15.3 network increases.

\section{CONCLUSIONS}

In this paper, we study the coexistence issue between IEEE 802.15 based Wireless Personal Area Network technologies. We present analysis on the channel collision probabilities with focuses on the coexistence scenarios between one WPAN technology with another. The results show that channel allocation conflicts occurs frequently in all cases, and is especially severe between an IEEE 802.15.3 and multiple IEEE 802.15.4 networks. On the other hand, the probability of nonconflict channel allocation is less dramatic between a single IEEE 802.15.1 and other coexisting IEEE 802.15.4 networks. Nonetheless, the proposed models in this paper are applicable to model coexistence issues between other wireless technologies (if channel allocation mechanism is known). Future work of this study is to take the error models (e.g. SNR vs PER, SNR vs distance, etc.) of each WPAN technology into account, and extend our analysis to model the packet/frame error rates for multiple coexisting WPAN networks.

\section{REFERENCES}

[1] "Bluetooth specifications v1.1," http://www.bluetooth.com.

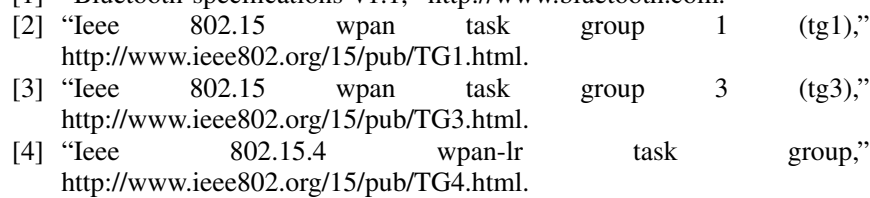

] "Ieee 802.11, the working group setting the standards for wireless lans," http://grouper.ieee.org/groups/802/11/.

[6] J. Lansford, A. Stephens, and R. Nevo, "Wi-fi (802.11b) and bluetooth: Enabling coexistence," IEEE Network, pp. 20-27, Sept/Oct 2001.

[7] S. Krishnamoorthy, M. Robert, S. Srikanteswara, M. Valenti, C. Anderson, and J. Reed, "Channel frame error rate for bluetooth in the presence of microwave ovens," in IEEE VTC Fall, 2002.

[8] N. Golmie, N. Chevrollier, and I. Elbakkouri, "Interference aware bluetooth packet scheduling," in GLOBECOM, 2001.

[9] N. Golmie, "Bluetooth dynamic scheduling and interference mitigation," ACM Mobile Networks and Applications (MONET), vol. 9, pp. 21-31, February 2004.

[10] A. Conti, D. Dardari, G. Pasolini, and O. Andrisano, "Bluetooth and ieee 802.11b coexistence: Analytical performance evaluation in fading channels," IEEE Journal on Selected Areas in Communications, vol. 21, no. 2, pp. 259-269, February 2003.

[11] I. Howitt and J. A. Gutierrez, "Ieee 802.15.4 low rate-wireless personal area network coexistence issues," in IEEE WCNC, 2003.

[12] "Ieee 802.15 wpan task group 2 (tg2)," http://www.ieee802.org/15/pub/TG2.html.

[13] P. Johansson, R. Kapoor, M. Kazantzidis, and M. Gerla, "Bluetooth: An enabler for personal area networking," IEEE Network Magazine, Sept/Oct 2001.

[14] "Bluetooth specifications v1.2," http://www.bluetooth.com.

[15] "Bluetooth specifications core v2.0," http://www.bluetooth.com.
[16] "Ieee 802.15 wpan high rate alternative phy task group 3a (tg3a)," http://www.ieee802.org/15/pub/TG3a.html.

[17] A. El-Hoiydi, "Interference between bluetooth networks - upper bound on the packet error rate," IEEE Communications Letters, vol. 5, no. 6, pp. 245-247, June 2001.

[18] "Dirichlet's box principle - from mathworld-: A wolfram web resource," http://mathworld.wolfram.com/DirichletsBoxPrinciple.html. 